University of Nebraska - Lincoln

DigitalCommons@University of Nebraska - Lincoln

Faculty Publications in the Biological Sciences

Papers in the Biological Sciences

$12-2006$

\title{
Identifying landscape scale patterns from individual scale processes
}

Andrew J. Tyre

University of Nebraska at Lincoln, atyre2@unl.edu

Brigitte Tenhumberg

University of Nebraska - Lincoln, btenhumberg2@unl.edu

C. Michael Bull

Flinders University of South Australia, michael.bull@flinders.edu.au

Follow this and additional works at: https://digitalcommons.unl.edu/bioscifacpub

Part of the Life Sciences Commons

Tyre, Andrew J.; Tenhumberg, Brigitte; and Bull, C. Michael, "Identifying landscape scale patterns from individual scale processes" (2006). Faculty Publications in the Biological Sciences. 118.

https://digitalcommons.unl.edu/bioscifacpub/118

This Article is brought to you for free and open access by the Papers in the Biological Sciences at DigitalCommons@University of Nebraska - Lincoln. It has been accepted for inclusion in Faculty Publications in the Biological Sciences by an authorized administrator of DigitalCommons@University of Nebraska - Lincoln. 
Published in Ecological Modelling 199:4 (December 16, 2006), pp. 442-450; doi: 10.1016/j.ecolmodel.2005.12.001

(Special issue on "Pattern and Processes of Dynamic Mosaic Landscapes -- Modelling, Simulation, and Implications")

Copyright (c) 2006 Elsevier B.V. Used by permission. http://www.sciencedirect.com/science/journal/03043800

Published online August 21, 2006.

\title{
Identifying landscape scale patterns from individual scale processes
}

\author{
Andrew J. Tyre, ${ }^{1}$ Brigitte Tenhumberg, ${ }^{2}$ and C. Michael Bull ${ }^{3}$ \\ 1. School of Natural Resources, University of Nebraska-Lincoln, Lincoln, NE 68583-0819, USA \\ 2. School of Biological Sciences and School of Natural Resources, University of Nebraska-Lincoln, Lincoln, NE 68588-0118, USA \\ 3. School of Biological Sciences, Flinders University of South Australia, Adelaide, SA 5005, Australia \\ Corresponding author - A. Tyre, tel 402 472-4054, fax 402 472-2946, email atyre2@unl.edu
}

\begin{abstract}
Extrapolating across scales is a critical problem in ecology. Explicit mechanistic models of ecological systems provide a bridge from measurements of processes at small and short scales to larger scales; spatial patterns at large scales can be used to test the outcomes of these models. However, it is necessary to identify patterns that are not dependent on initial conditions, because small scale initial conditions will not normally be measured at large scales. We examined one possible pattern that could meet these conditions, the relationship between mean and variance in abundance of a parasitic tick in an individual based model of a lizard tick interaction. We scaled discrepancies between the observed and simulated patterns with a transformation of the variance-covariance matrix of the observed pattern to objectively identify patterns that are "close".

The results indicate that it is possible to generate patterns that are independent of initial conditions, verifying that the small scale processes in the model are able to reproduce the large scale patterns observed in real data. The pattern analysis also indicates that we have a poor understanding of the density dependent effect of larval engorgement success and host refuge use.
\end{abstract}

Keywords: pattern based modeling, ectoparasite, host parasite interactions, Aponomma hydrosauri, Tiliqua rugosa

\section{Introduction}

Extrapolating across scales is a critical problem in ecology; we must predict the responses of ecosystems at large spatial and long temporal scales, but logistical constraints force us to measure and conduct experiments at much smaller and shorter scales. Measuring processes over long periods of time at large spatial scales is particularly difficult; it is possible to measure patterns, i.e. static snapshots at a single point in time, over large spatial extents. Explicit mechanistic models of ecological systems can provide a bridge from measurements of processes at small and short scales to larger scales; spatial patterns at large scales can be used to test the outcomes of these models. However, if there is significant feedback between the large scale pat- terns, and the small scale processes, we must have an accurate pattern to initialize the model with in order to predict a future large scale pattern. Ideally, we would verify model outputs without this sensitive dependence on initial conditions.

A promising recent development is the notion of "pattern based modeling" (Grimm et al., 1996), where the goal is to identify patterns in empirical data that can be used as filters on the model output to indirectly estimate parameters (Wiegand et al., 2003). In particular, if we identify patterns at large scales that are independent of initial spatial structure, then we can use these patterns to verify the model even if we are unable to accurately characterize the initial conditions of the model. The pattern analysis will also reveal significant gaps in our understanding of the system. 
The particular system of interest to us is the interaction between an ectoparasitic tick (Aponomma hydrosauri: Ixodidae) and its lizard host (Tiliqua rugosa: Scincidae) at Mt. Mary, South Australia (Tyre et al., 2003). This is part of a larger project examining the spatial and temporal dynamics of a parapatric boundary between $A$. hydrosauri and another ectoparasitic tick of Tiliqua, Amblyomma limbatum (Bull and Burzacott, 2001). Tyre et al. (2003) demonstrated (1) engorgement success of larval ticks is dependent on the number of ticks attempting to attach, and (2) at the landscape scale there is a positive relationship between the mean tick abundance per lizard, and the aggregation coefficient $k$ of a negative binomial distribution fitted to the number of ticks on lizards. Although the latter pattern is expected in the presence of density dependent engorgement (Pacala and Dobson, 1988), it is not clear whether the individual scale process is sufficient to generate the landscape scale pattern. The relationship between the mean and $k$ is also independent of any particular population trajectory, and thus it could serve to verify the structure of the model even though we cannot specify the initial distribution of ticks and hosts across the entire landscape.

In this paper, we develop an individual based, spatially explicit model which serves several purposes. First, it is a quantitative expression of our knowledge about the host/ parasite ecological system. Second, it allows us to experiment with alternative processes, and to formulate observations and experiments that will improve our knowledge of the system. Finally, it provides a vehicle for testing whether or not known individual scale processes lead to population and landscape scale patterns consistent with observations; this is our emphasis in this paper. First we describe the basic model structure and develop parameter estimates from existing empirical data. We then discuss the patterns we are interested in, and measures of discrepancy between simulated and observed patterns.

\section{Individual based, spatially explicit model}

Model parameters are given in italics and listed in Table 1. Chilton (1989) conducted extensive measurements of the response of life history parameters of $A$. hydrosauri, such as development time and survival, to temperature and humidity. In general, these experiments involved placing ticks on lizards or in vials, and then holding them under the appropriate conditions until the appropriate developmental process was complete. The time to engorge, develop, or die was recorded to the nearest day. In each specific section we describe how we used these data to obtain means, variances, and temperature effects for tick life history parameters.

From a tick's point of view, the landscape consists of the lizard hosts and their nocturnal refuge sites. There are numrefuges randomly distributed refuges in a $1 \mathrm{~km} \times 1 \mathrm{~km}$ square, used by numlizards lizards whose home ranges are centered on a randomly chosen refuge. All refuges within $100 \mathrm{~m}$ of the center refuge are included in the home range. Tyre (1999) calculated Jolly-Seber estimates of lizard density near Mt. Mary, South Australia (SA) from random capture data described in Bull (1995). Assuming that the random
Table 1. Parameters included in the sensitivity analysis, the distribution used to represent uncertainty, and the parameters of the uncertainty distributions

\begin{tabular}{llcc}
\hline Variable & Distribution & Location & Scale \\
\hline numrefuges & Uniform & 7500 & 12500 \\
numlizards & Uniform & 50 & 150 \\
devregg & Normal & 53.1 & 5.31 \\
devrlarvae & Normal & 21.9 & 2.19 \\
devrnymph & Normal & 28.4 & 2.84 \\
devradult & Normal & 26.5 & 2.65 \\
devvegg & Normal & 1.3 & 0.13 \\
devva & Normal & -10.1 & 3.03 \\
devvb & Normal & 4.2 & 1.26 \\
devtegg & Normal & 1.9 & 0.19 \\
engrlarvae & Normal & 30.6 & 3.06 \\
engrnymphs & Normal & 22.7 & 2.27 \\
engradults & Normal & 39 & 3.9 \\
engvlarvae & Normal & 11.7 & 1.17 \\
engvnymphs & Normal & 16.7 & 1.67 \\
engvadults & Normal & 17.6 & 1.76 \\
surrlarvae & Normal & 13.8 & 1.38 \\
surrnymphs & Uniform & 29.84 & 44.76 \\
surradults & Uniform & 80 & 120 \\
surva & Normal & -2.07 & 0.207 \\
survb & Normal & 1.67 & 0.167 \\
surta & Normal & 91.873 & 9.1873 \\
surtb & Normal & -0.091 & 0.0091 \\
devta & Normal & 18591 & 1859.1 \\
devtb & Normal & -2.1843 & \\
0.21843 & & & \\
tickeffect & (See text) & -0.002 & - \\
ticksuccess & (See text) & -0.243 & 0.3 \\
sampleefficiency & Uniform & 0.05 & \\
\hline Eor uniform & a & & \\
\hline
\end{tabular}

For uniform distributions the location and scale represent lower and upper limits. For normally distributed uncertainty the location is the mean, and the scale is the standard deviation.

capture transect is sampling lizards from a $400 \mathrm{~m}$ wide strip (based on $200 \mathrm{~m}$ diameter home ranges on either side of the transect), lizard density ranges from 15 to 420 lizards $/ \mathrm{km}^{2}$. The median lizard density is 100 lizards $/ \mathrm{km}^{2}$.

There are two time scales in the model. On the short time scale, movement of lizards, birth, development, and death of ticks is modeled each day. A series of days is then aggregated into a season, which is 210 days (1st September to 31st March) long. Development is frozen between seasons, under the assumption that autumn/winter temperatures are too low for tick activity. Ticks experience over-wintering mortality, and a simplified form of population dynamics among lizard hosts also takes place between seasons.

The choice of a single day as the basic time step is logical given the assumption that all significant movement of ticks on and off lizards occurs only in refuges entered at night. Ticks are adapted to detach in the nocturnal refuges of their hosts, where desiccation risks are lower, and the chances of finding another host are higher (Bull and Smyth, 1973). Boarding a lizard requires that a tick in the right life history state detects and moves toward a lizard that has entered a refuge. This movement ability is not great; some ticks were unsuccessful in boarding lizards even when confined together with a host in a calico bag for $48 \mathrm{~h}$ (Chilton, 1989). 
Within a single day, the model goes through several steps in the following order: ticks board lizards, lizards move between refuges, engorged ticks disembark from lizards, and ticks develop (Figure 1). We describe each step in more detail below.

\subsection{Tick embarkation, lizard movement, tick disembarkation}

At the beginning of a model day, all lizards are in the overnight refuges in which they spent the previous night. The model checks all ticks in lizard occupied refuges; ticks that are found to be in a suitable state (i.e. unfed larvae, nymphs, or adults) are moved onto the lizard. As noted above, assuming that all suitable ticks board lizards is almost certainly an overestimate. If there is more than one lizard in a particular refuge, the number of ticks boarding a lizard will be binomially distributed with $n$ trials (the number of ticks in the refuge in the correct life history stage) and probability of boarding $b$. For all lizards except the last lizard in the refuge, $b$ is the reciprocal of the number of lizards using the refuge that night. All remaining ticks in the refuge board the last lizard listed as being present in that refuge on that night. The position within the refuge list is random with respect to landscape location, and age class of lizard. As adult ticks board a lizard, the sex of each is determined randomly assuming a 1:1 sex ratio. The final process that occurs in this part of the model is to assign the number of days each tick will require to engorge as a normally distributed variable with a stage specific mean and standard deviation. This engorgement "index" is used during subsequent model days to determine when a tick has successfully engorged in the tick development step.

In the next step of the daily cycle, lizards move to new refuges. Each day, lizards move from one overnight refuge to another overnight refuge chosen randomly with equal probability from among those in their home range. Note that this means they can spend consecutive nights in a refuge, and more than one lizard can occupy a refuge overnight. This is the simplest scenario that can be implemented in the absence of information about the distribution of different types of refuges, the way in which neighboring refuges are utilized by individual lizards, and the way in which lizards may interact on contact with other lizards. We assume that individual lizards are capable of moving over their entire home range during the day, and that there is no systematic bias either toward or away from particular kinds of refuges, or from their previously occupied refuge.

The next step within the daily cycle is to drop off successfully engorged ticks into their new refuges. Essentially, ticks that are on lizards, which completed engorgement on the previous development step (i.e. the previous night), are dropped off in the new refuge chosen by their host lizard.

The final step of the daily cycle handles development and mortality of all ticks, regardless of their current location. During this step, each tick is checked to see whether it ages, survives, or lays eggs, depending on its current stage and whether it is on a lizard or not.

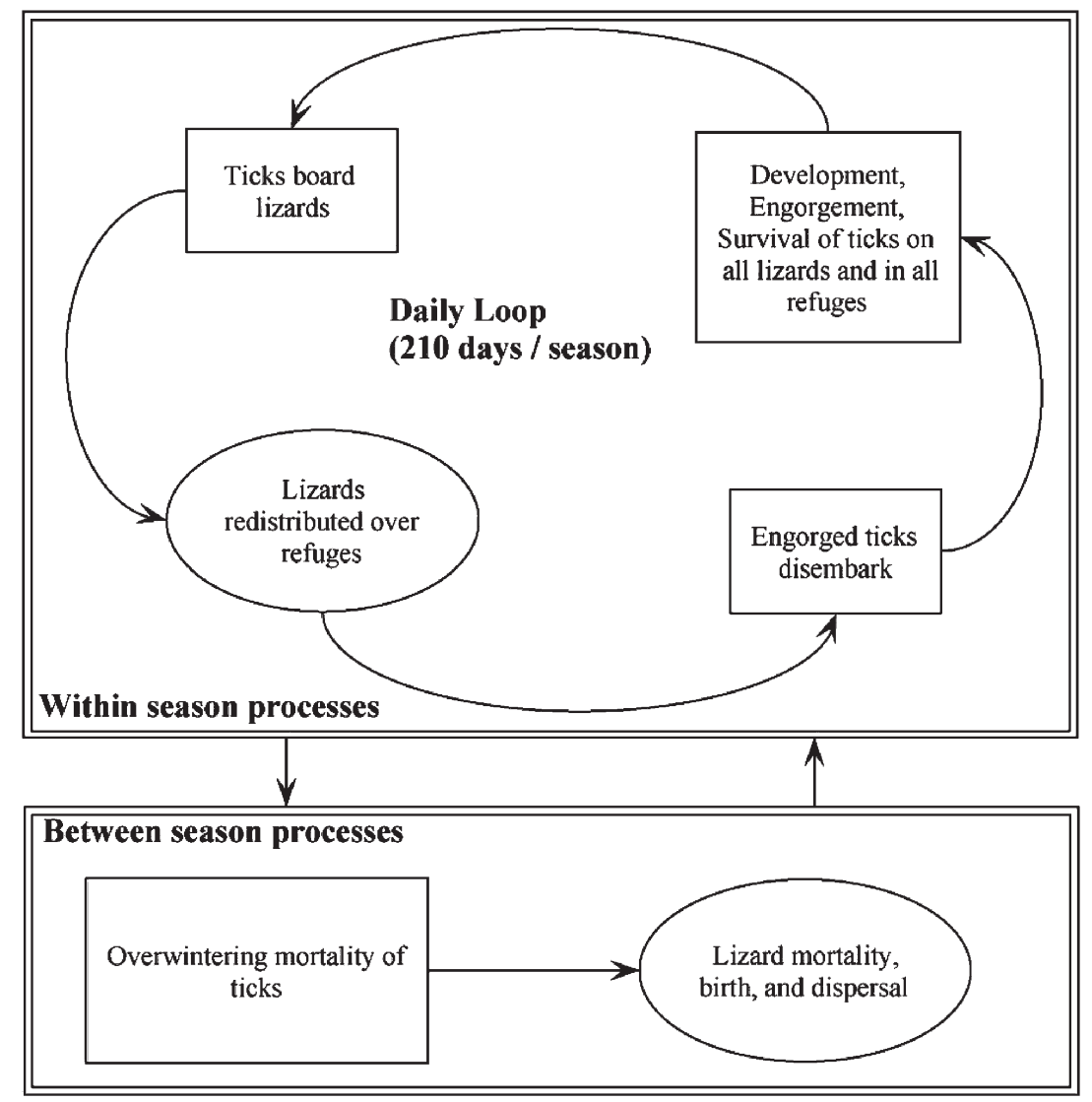

Figure 1. Flowchart of main model processes. Processes that are attributes of lizard population dynamics and behavior which indirectly affect the ticks are placed in ovals, while processes directly affecting ticks are in rectangles. 


\subsection{Growth and feeding}

Stages that are engaged in growth or feeding (eggs, engorged stages in refuges, and unfed stages on lizards) follow a threshold process, where each stage lasts for a fixed number of days for each individual. Each individual is assigned a normally distributed random number as a development or engorgement time on entry to a new life history stage. Both the mean and the variance are stage specific. For example, newly oviposited eggs have a hatching threshold with a mean of 53 days (devregg; Table 1) and a variance of 1.3 days (devvegg). During the daily development step, each individual tick has its development or engorgement index decreased by 1 day. On the day the index reaches 0 , the individual moves to the next stage (e.g. an egg hatches to an unfed larva, or a feeding nymph detaches). This means that the duration of all growing and feeding stages is normally distributed. This method is similar to those used for physiologically structured population models (Gurney et al., 1986), but includes variability between individuals. Chilton (1989) had good estimates for the average developmental times for all stages (devregg, devrlarvae, devrnymph, devradult); the variances were less consistent but increased with the mean developmental time according to $\sigma^{2}=$ devva $\cdot \mathrm{e}^{\text {devvb } \mu}$. We used this relationship to calculate the variance of developmental time for larvae, nymphs, and adults. The mean (engrlarvae, etc.) and variance (engvlarvae, etc.) of feeding periods was estimated directly from data for all stages.

Feeding has one additional complication. Not all individuals succeed in attaching, engorging and detaching, and this is one of the few places where density dependence is known to occur in the system. We calculated the probability of successfully engorging

$$
\begin{gathered}
\mathrm{p}\{\text { successful engorgement }\}=\frac{\mathrm{e}^{\beta}}{1+\mathrm{e}^{\beta}}, \\
\beta=-0.243-0.002 \text { (\# of ticks) }
\end{gathered}
$$

assuming that this probability depends on the total number of ticks of all stages at the time engorgement is complete (Tyre et al., 2003). The mechanism underlying the relationship between tick density and engorgement success is presently unknown, and this empirical relationship is the simplest to implement in the model. At present we assume density dependence applies to all tick stages equally.

\subsection{Survival}

Predation on ticks within refuges by other invertebrates does occur (Bull et al., 1988), but is both spatially and temporally unpredictable, and we do not include it in the current model. When a host lizard dies from predation (primarily automobiles near Mt. Mary) or old age all ticks on the lizard also die. We included this mortality in a single between season event (see below). We assume that the primary source of daily mortality for ticks is desiccation. The habitat has low rainfall (150-250 $\mathrm{mm}$ annually), and most development occurs during the hot, dry summer. The only moisture source available to ticks is a blood meal, and newly molted, unfed ticks must wait until another host ar- rives before they can replenish their moisture supply. Eggs, engorged ticks in refuges, and ticks feeding on lizards are assumed to be unaffected by desiccation. We modeled mortality similarly to development, by providing each individual with a normally distributed time to death. This is the number of days that each individual is expected to survive without feeding. The time is decreased by 1 day in each developmental step, and individuals that reach zero are killed. Death is presumed to have occurred as a result of higher temperatures during the day, and so mortality in a refuge precedes ticks boarding lizards that enter that refuge on the next day.

Chilton (1989) estimated the survival times for larvae (surrlarvae) under a wide range of temperatures and relative humidity. The average survival time of unfed nymphs (surrnymphs) and adults (surradults) are completely free parameters, but observations of nymphs surviving at hot dry conditions for 10-15 days suggest that survival times for both adults and nymphs are much longer than for larvae under normal conditions. Variance in survival times of larvae increased with the mean survival time $\left(\sigma^{2}=\right.$ surva $\cdot \mathrm{e}^{\text {survb } \mu}$; Table 1); we assumed the same relationship applied to variance in nymph and adult survival times.

\subsection{Mating and oviposition}

When an unfed adult tick boards a lizard, it is randomly assigned to be a male or female with a sex ratio of 1:1. Adult male ticks remain on lizards for the remainder of their life, assumed to be a fixed 180 days. The only contribution they have beyond this point is to mate with unfed female ticks. After boarding a lizard there is a fixed 5 day period before an adult male is mature and capable of mating. When an unfed female boards a lizard, if there is one or more mature males aboard she is mated immediately. Otherwise, she waits on that lizard until a mature male appears, or 180 days passes. There is no negative impact of waiting to mate on a females subsequent reproductive output, although a negative impact has been observed in laboratory experiments (Chilton, 1989). Once a female is mated, then she begins to engorge as described above for all other stages. This does introduce a slight Allee effect through delaying reproduction by females that board lizards without males.

Adult female ticks that have mated, successfully engorged, and dropped off in a refuge enter a pre-oviposition stage, the duration of which is normally distributed and handled identically to aging, feeding, and molting. After pre-oviposition the number of eggs oviposited by a female tick per day depends on age; it peaks at 100 eggs/day at 10 days of age and all oviposition ceases by 40 days of age. We used laboratory observations of average oviposition rates rounded down to the nearest integer. There is no stochasticity in oviposition.

\subsection{Between season processes}

There are two processes that occur between the end of one season and the beginning of the next: over-wintering tick mortality and lizard population dynamics. All ticks, regardless of location, have a stage specific chance of mortality 
over winter. This reflects exposure, desiccation, disease, predation, and fungal infection. We set this to $10 \%$ for all stages other than eggs. It is set low relative to mortality during the active season because the risk of desiccation is reduced in the cooler, wetter climate of winter, and invertebrate predators are less active. However, laboratory observations indicate that no eggs hatch when held at temperatures of less than $15^{\circ} \mathrm{C}$. Therefore, egg mortality is $95 \%$ over the winter in the model, which allows for a small margin of error in the laboratory estimate of $100 \%$.

Lizard population dynamics is also simplified. A flat $10 \%$ of all lizards are chosen at random and killed at the end of each season. Empirical observations place annual adult survival at around $90 \%$ (Bull, 1995). Any ticks on the killed lizards are likewise killed. The killed lizards are replaced from newborns whose mothers are chosen at random from the surviving lizards. These newborn lizards spend one season in their mother's home range, before randomly choosing a home range of their own (natal dispersal). This results in no net change in the number of lizards available, but tends to redistribute $10 \%$ of the population to new locations each season after the first two.

\subsection{Temperature effects}

We used 31 years (1967-1998) of daily minimum and maximum temperatures measured at Eudunda, SA approximately $30 \mathrm{~km} \mathrm{SW}$ of the study area to drive weather variation in the model. The maximum temperatures in Eudunda in 1997 are highly correlated with the average daily temperature measured in bluebush refuges at the study site $\left(T_{\text {avg }}=3.4057+0.6641 T_{\max } R^{2}=0.87, F_{1,194}=1345\right.$, $p \ll 0.001$ ). We used this regression equation to predict average daily temperatures at the study site for 31 seasons. We use these temperatures to generate sequences of random seasons drawn with replacement from this set. The sequence of days within each season is used unmodified in order to include the effect of small scale correlations that are present in the weather. We defined five temperature categories $\left(T<15{ }^{\circ} \mathrm{C} ; 15^{\circ} \mathrm{C} \leq \mathrm{T}<20^{\circ} \mathrm{C} ; 20^{\circ} \mathrm{C} \leq \mathrm{T}<25^{\circ} \mathrm{C}\right.$; $25^{\circ} \mathrm{C} \leq \mathrm{T}<30^{\circ} \mathrm{C} ; 30^{\circ} \mathrm{C} \leq \mathrm{T}$ ) based on the availability of information about the response of survival and development to temperature (see below).

Temperature has a strong effect on average development and survival times of ticks. We assumed that engorgement rates were unaffected by temperature because host lizards behaviorally thermoregulate, creating a constant temperature environment for engorgement. Chilton (1989) observed mean developmental time for larvae decreasing with temperature $\mu=$ devta $\cdot \mathrm{e}^{\text {devtbt }}$. Similarly, mean survival times for larvae decreased exponentially with increasing temperature $\mu=\operatorname{surta} \cdot \mathrm{e}^{\mathrm{surtb} \cdot t}$. There are nearly as many ways of incorporating such variability in models of arthropod dynamics as there are models. For example, Logan et al. (1976) discussed an analytic model that allows non-linear changes in rate with temperature and is therefore superior to the degree-day concept which is inherently linear. Unfortunately the Logan et al. (1976) method relies on data across a wider range of temperatures than have been measured for ticks. We chose to discretize temperature into the five classes introduced above.
We assumed that temperature acts by increasing or decreasing the relative development or mortality rate. We calculated this relative effect for each of the five temperature classes by dividing the average time needed at the midpoint of the temperature class by that needed at the baseline temperature. The relative temperature effects calculated for larvae were also applied to nymphs and adults.

The relative effect of temperature on egg development was directly estimated from observations in Chilton (1989). Below $20{ }^{\circ} \mathrm{C}$ no development occurs in eggs, while development rate is devtegg faster when temperatures are $25{ }^{\circ} \mathrm{C}$ or greater.

\subsection{Pattern comparison}

The observed pattern is the relationship between the mean and aggregation coefficient of a negative binomial distribution describing the among lizard distribution of larvae, nymphs, and adults (Tyre et al., 2003), summarized as the intercept and slope of a linear regression among years between 1983 and 1997. We sample model output with a virtual ecologist (Tyre, 1999) along a simulated transect through the center of the landscape once each simulated week. The virtual ecologist encounters lizards whose home range intersects the transect with probability sampleefficiency; encountered lizards have all their ticks enumerated by stage. For each simulated year in which at least three lizards were captured, we calculated the average number of ticks of each stage, and the aggregation coefficient using the moment estimator:

$$
k=\frac{\mu^{2}}{\mu-\sigma^{2}}
$$

where $\mu$ is the mean and $\sigma^{2}$ is the variance of the among lizard distribution for that year. All simulated years for a given parameter combination were then subjected to a linear regression to estimate the intercept and slope of the relationship between $k$ and $\mu$. The unscaled discrepancy $D$ for a given pattern and parameter combination is simply the Euclidean distance between the observed intercept (a) and slope $(b)$, and the simulated intercept and slope

$$
D=\sqrt{(a-\tilde{a})^{2}+(b-\tilde{b})^{2}}
$$

These unscaled discrepancies do not account for the fact that our target pattern is not precisely observed; $a$ and $b$ are also estimates, and the uncertainty in these estimates is described with a variance-covariance matrix $\mathbf{V}$. We chose to scale the discrepancies by the magnitude of a $95 \%$ confidence ellipse around our target pattern

$$
\Pi=\left(f^{-1} \mathbf{P}\right) \mathbf{L}^{-1}
$$

where $\mathbf{P}$ is a matrix of the observed and simulated patterns, $f$ the 95th quantile from an $\mathrm{F}$ distribution with the appropriate degrees of freedom, and $\mathrm{L}^{-1}$ is the inverse of the Cholesky decomposition of the variance-covariance matrix $\mathbf{V}$. This transformation of the coordinate system converts the $95 \%$ confidence ellipse into the unit circle. $\Pi$ is then a matrix of the scaled patterns; the scaled discrepancies $\Delta$ are the Euclidean distances between the scaled observed pattern and each scaled simulated pattern. If the scaled discrepancy for a parameter combination is less than one, it falls inside the 


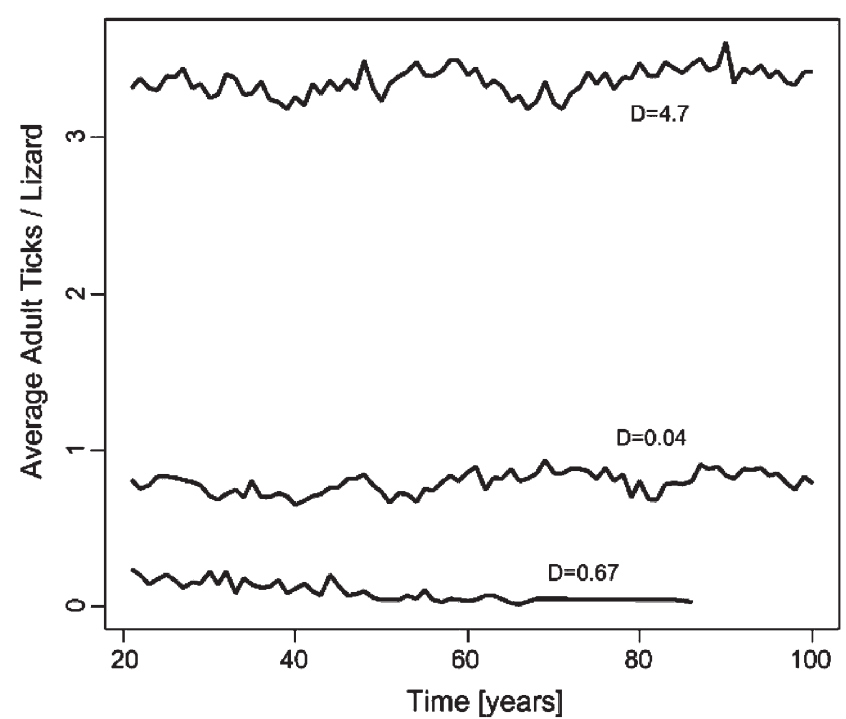

Figure 2. Average adult ticks per lizard, conditional on a replicate not being extinct, over time for three separate parameter combinations with the largest, smallest, and an intermediate pattern discrepancy $(D)$.

95\% confidence ellipse around the observed pattern; if the scaled discrepancy is larger than one it falls outside the confidence ellipse.

In our case, we have three observed patterns - the relationship between the mean and $k$ for larvae, nymphs, and adults. We first examine the discrepancies for each pattern individually. Then we combine the discrepancies using the geometric mean, because the discrepancies are scaled such that one is the dividing line for "close". The geometric mean places greater weight on small discrepancies.

\subsection{Sensitivity analysis}

We conducted the sensitivity analysis of the model on the 28 parameters by forming a Latin hypercube (Blower and Dowlatabadi, 1994; Tenhumberg et al., 2004). Briefly, this approach assumes that uncertainty in a parameter follows a specific probability distribution. Each distribution is divided into K equi-probable segments. A set of parameters for a single run of the model is created by sampling these distributions without replacement; this results in $K$ unique parameter combinations. We set $K=100$ for this initial test of the model; this is well over the empirical rule of $K>4 / 3$ the number of parameters for Latin hypercube sensitivity analysis (Mckay et al., 1979). Each parameter combination was run 100 times - runs that went extinct before year 20 were discarded. While the number of parameterizations is relatively small, our intent was to explore the potential utility of these patterns, and provide initial data to determine the number of years and replicate runs needed for simulated patterns to stabilize. This information will be used to refine future tests of this model with additional data.

In general we assumed parameter uncertainty followed normal distributions where we had estimated parameters from real data. We used the estimated standard errors to calculate the variance of each parameter distribution, but maintained a minimum coefficient of variation of $10 \%$ in each

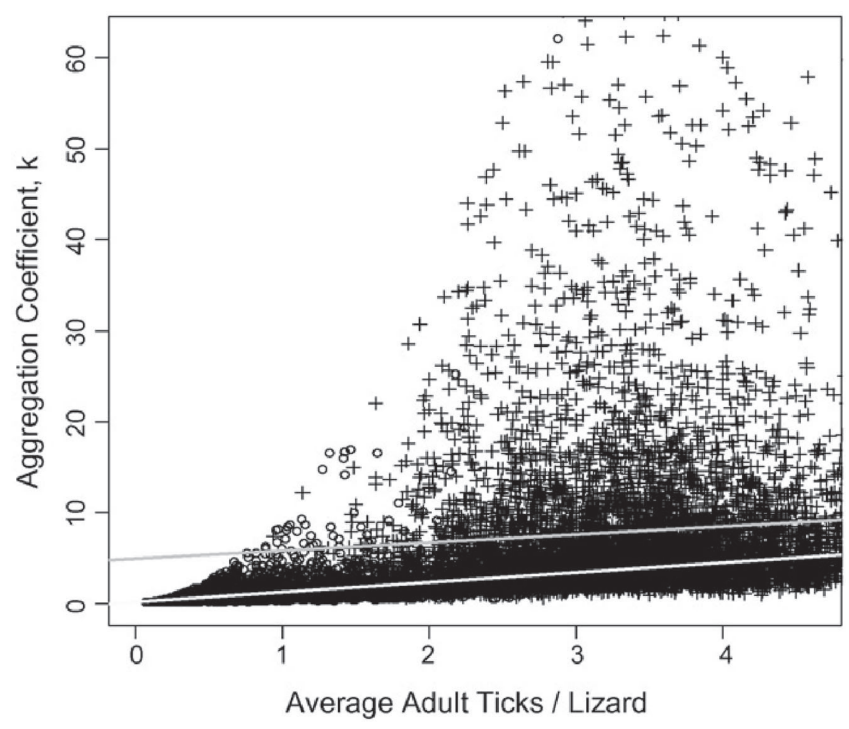

Figure 3. Simulated relationship between the mean abundance of ticks and the aggregation coefficient $k$ for two runs, one with the lowest observed pattern discrepancy (circles), and with the highest observed pattern discrepancy (pluses); there are 100 replicates of each run. The white line is the observed linear regression (the target pattern), and the grey line is the simulated pattern for the run with the highest discrepancy. The regression line for the run with the lowest discrepancy is not visibly distinguishable from the white line.

parameter. For parameters that were largely guesswork (e.g. lizard and refuge densities), we used uniform distributions across a broad range of reasonable parameters. With one exception we assumed all parameters were varying independently.

The exception was the density dependence parameters; bootstrap analysis of the regression model indicated that the two parameters were highly non-normally distributed and correlated (Tyre et al., 2003). We sampled the distribution of 5000 bootstrap replicates without replacement to produce the $K$ combinations incorporating the observed correlations between the two parameters.

\section{Results}

For all results we discarded the first 20 years of a simulation run to enable the population to settle into a quasi-stationary distribution; inspection of time series plots for extant population size indicated that 20 years was sufficient for populations that were not rapidly going extinct (Figure 2). In addition, populations that stabilize at different levels have different pattern discrepancies, suggesting that our proposed patterns do reflect the quasi-stationary population structure. This is further confirmed by examination of scatterplots of the aggregation coefficients against the mean tick abundance for the runs with the lowest and highest discrepancy (Figure 3).

Changing the parameters of the model in the Latin hypercube leads to substantial variation in the simulated mean- $k$ relationship (Figure 4). The unscaled discrepancies $D$ (3) are the distances between a point representing a simulation run 


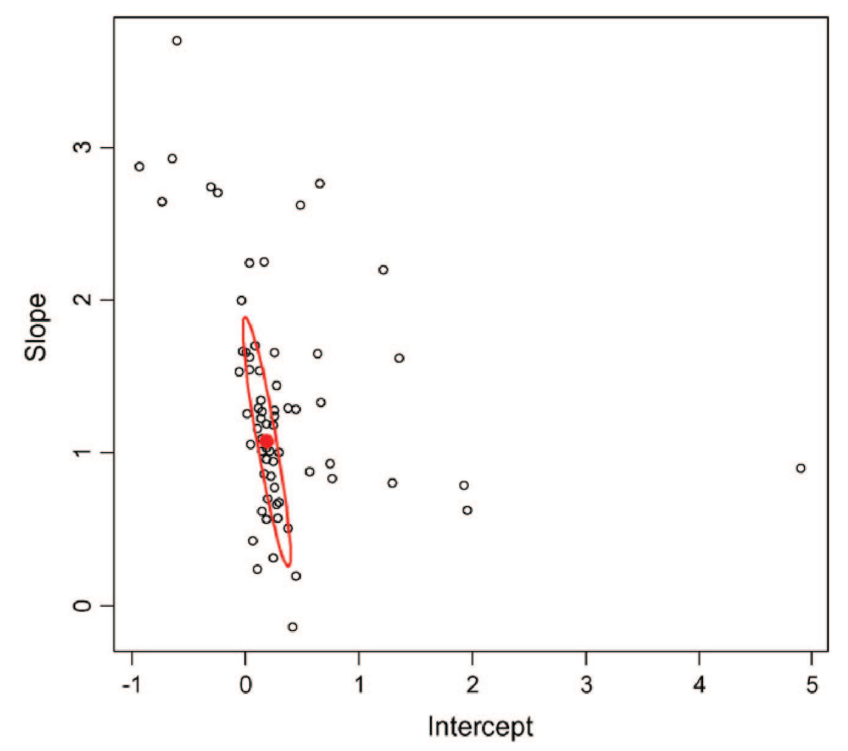

Figure 4. Scatterplot of intercept and slope of simulated mean- $k$ relationships for adult ticks. Observed mean- $k$ relationship indicated with solid circle and estimated $95 \%$ confidence ellipse indicated as a solid line. and the observed point on this scatterplot. There are 24 parameter combinations that are "close" to the observed pattern, in the sense that they are inside the 95\% confidence ellipse. The shape of the confidence ellipse indicates that the slope of the relationship is less well characterized than the intercept; hence in the pattern comparison it is important to rescale the discrepancies to give greater weight to differences in the intercept. The simulation best fits the adult pattern; only one and two runs are close to the patterns for larvae and nymphs, respectively (figures not shown). These results indicate that the model is capable of reproducing the observed pattern even with a relatively small number of trial parameter combinations.

Plotting the rescaled and combined discrepancies against each parameter in the Latin hypercube reveals the impact of a parameter on the simulated patterns (Figure 5); we show only the three parameters of greatest interest to us, and one example of a parameter with little apparent effect (average duration of the egg stage). Note that the discrepancy values are the same on every graph; what changes is the distribution of those discrepancies with respect to the parameter plotted on the $x$ axis. The two parameters describing the magnitude of density dependence in larval engorgement
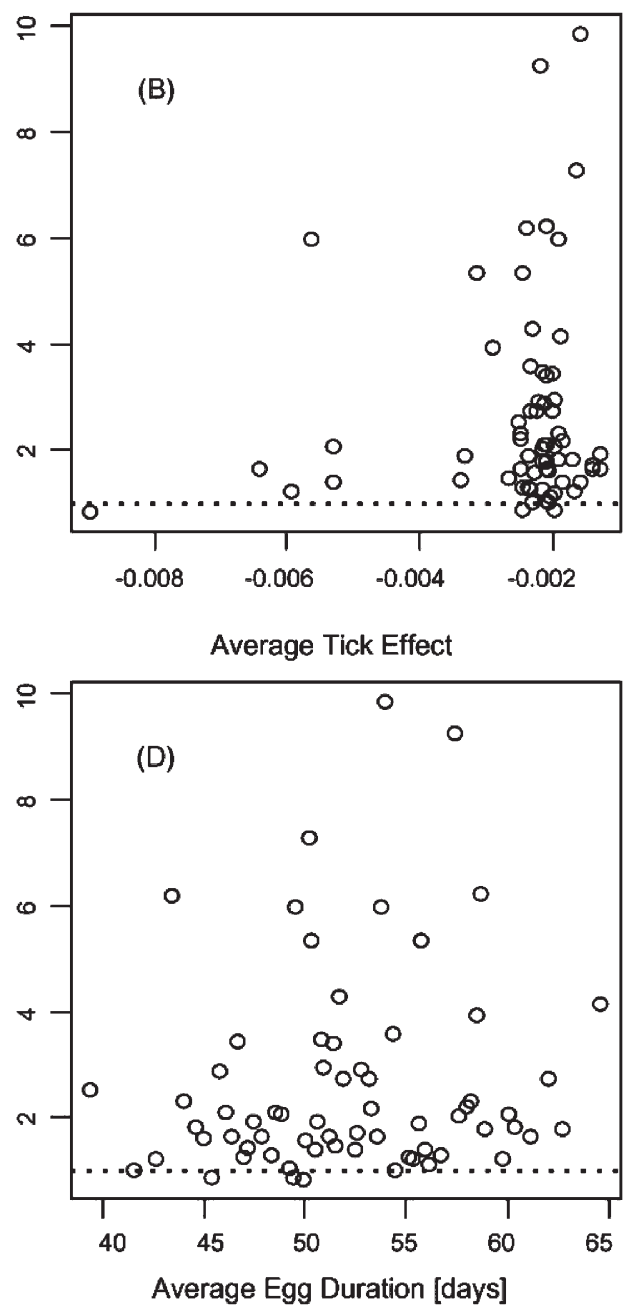

Figure 5. Scatterplots of scaled combined discrepancies against a selection of input parameters. Horizontal dotted lines indicate $\Delta^{*}=1$; points below this line are on average within the confidence ellipses of the observed patterns. 
success have the lowest discrepancy at extreme values of both parameters. Therefore, the density effect estimated in the laboratory likely underestimates the strength of this process in the field. The large scatter in these relationships is indicative of interactions between these parameters and other parameters in the model. Similarly, there is a strong relationship between the discrepancy and the ratio of lizards to refuge sites. High host populations relative to the available refuge sites are inconsistent with the observed patterns. There is a suggestion that the relationship has an intermediate optimum, with very low relative lizard numbers leading to an increase in the pattern discrepancies.

\section{Discussion}

Our primary goal was to determine if our model could recreate landscape level patterns given only information about individual level biology. In particular, we were interested in patterns that are independent of any particular set of initial conditions, as these are unknowable in the current situation. We successfully found some parameterizations that replicated a landscape level pattern of an increase in the aggregation coefficient with increasing mean abundance of ticks. This pattern is independent of particular initial conditions on the landscape, which provides great promise for analyzing this system despite limited empirical data of landscape distributions. Furthermore, we generated these patterns based only on biological knowledge of individual level life history and interactions.

While the current pattern analysis indicates that the model can replicate large scale patterns, there are some limitations. First, we used a moment estimator of the aggregation coefficient $k$, instead of the superior maximum likelihood estimator used with the observed data (Tyre et al., 2003). This was a convenient shortcut to deal with the high volume of data generated by the simulation, but could be contributing to variation in the simulated patterns. In addition, because the simulation was using a small piece of a landscape $(1 \mathrm{~km} \times 1 \mathrm{~km})$, the mean and $k$ tended to be based on smaller sample sizes in each year than was true for the observed data. This created occasional outliers - low mean, high variance points - that could have obscured some patterns.

It is intriguing that the pattern analysis performed worse on the larval and nymph patterns. This result shows that the adult pattern is easier to generate than the pattern for nymphs and larvae. One reason for this could be that the parameter ranges for processes acting on nymphs and larvae are wider relative to the "true range" than for adults and that therefore the probability to find a parameterization inside this range is lower. By expanding the number of parameterizations, say to 10,000 , we may find some 100 parameterizations which fit the pattern for nymphs $(\sim 1 \%$, the rate of matching for the current set), and this would provide an avenue to investigate how these patterns are different. Alternatively, the understanding of the biology of nymphs and larvae could be incomplete. In the real data the negative binomial distribution did not always provide a good representation of the data (Tyre et al., 2003). We postulate that this arises because of high correlations between larvae boarding lizards in refuges where a batch of eggs has recently hatched.

Increasing the number of parameterizations tested would allow greater exploration of combined patterns as described above, and also provide better information on interactions between parameters. This is because there will be a greater number of samples from each region of the parameter space. The primary barrier to increasing the number of parameterizations is computation time; a single replication of 100 years on a desktop workstation takes over an hour. The current set of runs took over 40 days to complete-a 100-fold increase in the number of parameterizations is not practical without using a distributed computer system. However, the initial set of runs demonstrated that the population reached a quasi-stationary distribution in about a fifth of the simulated time period. Thus, we could use the existing simulation data to determine how many simulated years, and how many replicated runs are required for the simulated patterns to stabilize. Once the estimated patterns have stabilized, additional simulated years or replicates are not providing additional information. Reducing the number of replicates and/or years would allow a greater number of parameterizations to be tested.

The effect of lizard abundance relative to refuge density on the pattern discrepancy suggests characterization of host abundance and refuge use is critical. We know that all bluebush refuge sites are not equal; lizards prefer bluebushes with a dome shape over bushes with foliage that does not reach the ground (Kerr et al., 2003). Lizards also shift usage towards different types of refuges throughout the season. The estimated density of bluebushes in the field is much larger than the refuge densities used in the simulation results shown here. Lizard abundance also varies across the landscape, although the exact pattern of variation has not been characterized. Using the median lizard density of 100 lizards $/ \mathrm{km}^{2}$, the lowest overall discrepancy implies a refuge density of 10,000 refuges $/ \mathrm{km}^{2}$, substantially lower than our current best estimates of overnight refuge site numbers.

Modelers often comment that models can be used to identify parameters or processes that are inadequately quantified. There are at least two examples of this in the present model. First, we found that the density dependent engorgement process is insufficiently understood; the lowest observed discrepancy was at extreme values of both density dependent parameters. Density dependent engorgement has only been measured over a range of densities that are extremely uncommon in the field. It is not known whether the same mechanism applies during other engorging stages. For example, if engorgement success is very high $(\approx 1)$ with fewer than 10 ticks per lizard and drops off sharply to the levels observed in the experiment, then this mechanism could lead to population regulation. Improved engorgement success at low densities would tend to raise the average population growth rates, while forcing these to drop off to the levels observed in the current model when densities increased (i.e. to strongly negative values).

Many things have been left out of the model: predation in refuges, crushing mortality during engorgement, high temperature mortality, less than $100 \%$ boarding rates. This is always a dilemma in modeling that the model is a gross simplification of reality. However, our approach allows us 
to test whether including some of these hypotheses will actually improve the ability of the model to generate the observed patterns. If not, the data do not justify inclusion of more detail. All of the processes mentioned would increase the mortality of ticks, and therefore increase the rate at which populations go extinct. Thus, we are left with the conclusion that lizards must not use all refuges equally. The only process that could increase the survival of ticks waiting in refuges for lizards is non-random use of the available refuges, i.e. if there are some refuges lizards are more likely to visit than others. If neighboring lizards tend to use the same criteria to pick refuges, this will then also increase the rate of horizontal transmission between lizards. Otherwise, non-random use within a home range, but not between home ranges (i.e. lizards do not know each other's choice criteria), will increase survival but not transmission. Correlated movement of mates will not increase transmission across the landscape by much, because the home ranges of mates overlap to a large degree even outside the mating season.

An obvious extension of this work is to explore other patterns in the data. The joint spatial-temporal correlation (Henebry, 1995) is one interesting possibility reflecting more of the spatial and temporal structure of the data while retaining independence from any particular trajectory. Simulating this pattern would require a much larger landscape than we used here. A second possibility is the relationship between the population growth rate, $\ln \left(N_{t+1} / N_{t}\right)$, and population size - this will be negative and increase in strength with density dependence. Finally, the incidence and intensity of tick infections at the end of a simulation run - averaged over a few years - would provide an additional pattern that is independent of initial conditions. This last would only work in the absence of a trend in the observed incidence and intensity.

In summary, pattern based modeling shows considerable promise for validating ecological models when the initial conditions of the model are poorly known or unobtainable. This kind of approach is critical when scaling up from individual level processes to landscape scale observations.

\section{Acknowledgments}

This work is supported by an Australian Research Council Discovery to CMB and AJT, and many previous Australian Research Council grants to CMB. Early development of the ideas in this paper was stimulated by discussions with Thorsten Wiegand during a visit to the Umweltforschung Zentrum, Leipzig, in 2001.

\section{References}

Blower and Dowlatabadi, 1994 S. M. Blower and H. Dowlatabadi, Sensitivity and uncertainty analysis of complex-models of disease transmission-An HIV Model, as an example, Int. Stat. Rev. 62 (1994), pp. 229-243.

Bull, 1995 C. M. Bull, Population ecology of the sleepy lizard, Tiliqua rugosa, at Mt. Mary, South Australia, Aust. J. Ecol. 20 (1995), pp. 393-402.

Bull and Burzacott, 2001 C. M. Bull and D. Burzacott, Temporal and spatial dynamics of a parapatric boundary between two Australian reptile ticks, Mol. Ecol. 10 (2001), pp. 639-648.

Bull et al., $1988 \longrightarrow$ C. M. Bull, N. B. Chilton, and R. D. Sharrad, Risk of predation for two reptile tick species, Exp. Appl. Acarology 5 (1988), pp. 93-100.

Bull and Smyth, 1973 C. M. Bull and M. Smyth, The distribution of three species of reptile ticks, Aponomma hydrosauri (Denny), Amblyomma albolimbatum Neumann, and Amb. limbatum Neumann. II. Water balance of nymphs and adults in relation to distribution, Aust. J. Zool. 21 (1973), pp. 103-110.

Chilton, 1989 N. B. Chilton In: C. M. Bull, editor, Life Cycle Adaptations and their Implications in the Distribution of Two Parapatric Species of Tick, Flinders University, South Australia (1989).

Grimm et al., 1996 V. Grimm, K. Frank, F. Jeltsch, R. Brandl, J. Uchmanski, and C. Wissel, Pattern-oriented modelling in population ecology, Sci. Total Environ. 183 (1996), pp. 151-166.

Gurney et al., 1986 W. S. C. Gurney, R. M. Nisbet, and S. P. Blythe, The systematic formulation of models of stage-structured populations. In: J. A. J. Metz and O. Diekmann, editors, The Dynamics of Physiologically Structured Populations, SpringerVerlag, Heidelberg (1986).

Henebry, 1995 - G. M. Henebry, Spatial model error analysis using autocorrelation indexes, Ecol. Modell. 82 (1995), pp. 75-91.

Kerr et al., 2003 - G. D. Kerr, C. M. Bull, and D. Burzacott, Refuge sites used by the scincid lizard Tiliqua rugosa, Austral Ecol. 28 (2003), pp. 152-160.

Logan et al., 1976 J. A. Logan, D. J. Wollkind, S. C. Hoyt, and L. K. Tanigoshi, An analytic model for description of temperature dependent rate phenomena in arthropods, Environ. Entomol. 5 (1976), pp. 1133-1140.

Mckay et al., 1979 M. Mckay, W. Conover, and R. Beckman, A comparison of three methods for selecting values of input variables in the analysis of output from a computer code, Technometrics 21 (1979), pp. 239-245.

Pacala and Dobson, 1988 S. W. Pacala and A. P. Dobson, The relation between the number of parasites host and host age-population-Dynamic causes and maximum-likelihood estimation, Parasitology 96 (1988), pp. 197-210.

Tenhumberg et al., $2004 \rightarrow$ B. Tenhumberg, A. J. Tyre, A. Pople, and H. P. Possingham, Do harvest refuges buffer Kangaroos against evolutionary responses to selective harvesting, Ecology 85 (2004), pp. 2003-2017.

Tyre, 1999 A. J. Tyre, Spatial Population Dynamics: Enter the Virtual Ecologist, Ph. D. Dissertation, University of Adelaide, Adelaide, South Australia, 1999.

Tyre et al., 2003 A. J. Tyre, C. M. Bull, B. Tenhumberg, and N. B. Chilton, Indirect evidence of density-dependent population regulation in Aponomma hydrosauri (Acari: Ixodidae), an ectoparasite of reptiles, Austral Ecol. 28 (2003), pp. 196-203.

Wiegand et al., $2003-\mathrm{T}$. Wiegand, F. Jeltsch, I. Hanski, and V. Grimm, Using pattern-oriented modeling for revealing hidden information: A key for reconciling ecological theory and application, Oikos 100 (2003), pp. 209-222. 